\title{
Heinz Holligers Streifzüge mit Robert Walser
}

\author{
Maskierungen
}

Für die musikalische Walser-Rezeption ist der Schweizer Oboist, Komponist und Dirigent Heinz Holliger ( $\left.{ }^{*} 1939\right)$ von zentraler Bedeutung. Mit dem Liederzyklus Beiseit (1990/91) setzte sich erstmals ein international bekannter Repräsentant der Neuen Musik mit dem Schweizer Schriftsteller auseinander. Robert Walser ruft bei Holliger eine neue Form >biografisch geprägten Komponierens hervor, bei dem die musikalischen Einfälle aus einer starken und zuweilen experimentell erzeugten Identifikation mit dem Leben und Schaffen des Dichters entstehen. Bei der Oper Schneewittchen (1997/98) nach Robert Walsers gleichnamigem Märchenspiel hat Holliger in seinem Kompositionsprozess diesen subjektiven und autobiografischen Aspekt noch verstärkt. ${ }^{1}$

\subsection{Einleitung}

Heinz Holligers Zyklus Beiseit entstand in der Zeit des Mauerfalls und der Öffnung des Eisernen Vorhangs: Innert weniger Monate lösten sich nicht nur die bisherigen Machtblöcke auf, auch die Wertesysteme zerfielen, ohne dass neue gesellschaftspolitische Ziele vorhanden gewesen wären (vgl. Kap. 8.1). Der die Orientierungslosigkeit beschreibende Robert Walser passte deshalb gut in diese Epoche. Robert Walser bekommt in jenen Jahren aber auch rein literarisch viel Aufmerksamkeit: Die von Bernhard Echte und Werner Morlang

1 Seit 1993 schreibe ich auf Wunsch von Heinz Holliger regelmäßig Konzerteinführungen, CDTexte und Aufsätze vor allem zu jenen Werken, in denen Holliger sich auf Literatur bezieht (vgl. Brotbeck: Komponierte Erkaltung). Solchen Arbeiten gehen oft mehrstündige Besuche und Telefongespräche voraus. Viele Informationen dieses Kapitels beruhen auf diesen informellen Treffen. Gewisse Teile basieren auf meinen Aufsätzen Scardanelli et l'/d'après Scardanelli (deutsch 1996, französisch 2007), Schneewittchen (französisch 2007) und anderen. Thomas Wördehoff bezeichnet sie im Falle von Schneewittchen als »betörenden Aberwitz« (Wördehoff: Lauter leise, linde Brisen, S. 47). Neue Erkenntnisse zum Verhältnis von Heinz Holliger zu Robert Walser bietet die von Heidy Zimmermann anlässlich der Basler Produktion von Schneewittchen herausgegebene Schrift Holligers Walser, in der ein Gespräch von Heidy Zimmermann mit Heinz Holliger abgedruckt ist, aus dem im Folgenden oft zitiert wird. 
seit 1985 herausgegebenen Bände mit den Mikrogrammen, jener in Kleinstschrift beschriebenen Blätter und Zettel, lösen ein großes Echo aus; der immer schon von Mythen umrankte Dichter erscheint damit noch um vieles rätselhafter.

In der europäischen und insbesondere der deutschen Musikgeschichte markiert der Mauerfall auch das definitive Ende der musikalischen Avantgarde und ihres materialästhetischen Diskurses. Ihr stolzes Selbstverständnis, den vordersten Stand der einen - im emphatischen Singular gedachten - Musikgeschichte zu repräsentieren, war nicht länger zu halten. Theodor W. Adorno hat dieses Avantgarde-Modell hauptverantwortlich konstruiert und geschichtsphilosophisch unterlegt. Stark verkürzend zusammengefasst: Je konsequenter sich die Neue Musik auf ihre Autonomie beschränkt und - dem Fortschritt verpflichtet - die immanent-logische Entwicklung des musikalischen Materials und die strukturelle Komplexität vorantreibt, desto höher ist gemäß Adorno ihr ästhetischer Wahrheitsgehalt einzuschätzen. Wichtig ist für Adorno in diesem Zusammenhang die radikale Ablehnung von Jazz, Unterhaltungsmusik und all ihrer Derivate, die er schon in den 193oer-Jahren als barbarisch denunzierte. ${ }^{2}$

So eurozentristisch die Konstruktion der Avantgarde im Sinne Adornos ist, so kreativ und wirksam hatte sie sich doch auf das Komponieren ausgewirkt, denn sie machte Mut, ohne Rücksicht auf gesellschaftlichen Common Sense Innovationen zu suchen und zu wagen. Das lässt sich gerade an Heinz Holligers künstlerischer Entwicklung exemplarisch verfolgen. Zwar zählt er mit Jahrgang 1939 nicht mehr zur Gründergeneration der Avantgarde nach 1945, aber er setzte sich in seinem interpretatorischen und kompositorischen Schaffen intensiv mit der Avantgarde auseinander und brachte sie als Oboist maßgeblich voran: über 200 Kompositionen, die für ihn und oft auch für seine Frau, die Harfenistin Ursula Holliger (1937-2014), geschrieben wurden, hat er uraufgeführt. Die Oboe ist dank Holliger ein anderes Instrument geworden, nicht etwa, weil das Instrument neu konstruiert oder umgebaut worden wäre, sondern weil Holliger als Interpret dessen Spieltechniken enorm erweiterte und damit die Oboe in ähnlicher Weise neu definierte, wie dies im 19. Jahrhundert Niccolò Paganini mit der Geige und Frédéric Chopin oder Franz Liszt mit dem Klavier getan hatten.

2 In seiner Verachtung des Jazz geht Adorno schon 1933 so weit, dass er das nationalsozialistische Verbot, im Rundfunk Jazz zu spielen, ausdrücklich begrüßt: »Die Verordnung, die es dem Rundfunk verwehrt, >Negerjazz zu übertragen, hat vielleicht einen neuen Rechtszustand geschaffen - künstlerisch aber nur durchs drastische Verdikt bestätigt, was längst entschieden ist: das Ende des Jazz.« Adorno: Abschied vom Jazz, S. 795. 
Wichtiger Katalysator dieser Entwicklung war Pierre Boulez, der von 1960 bis 1962 auf Einladung des Dirigenten und Mäzens Paul Sacher kurze Zeit an der Musik-Akademie Basel, deren Direktor Sacher damals war, Komposition unterrichtete und eine nicht unerhebliche Anzahl von jungen Schweizer Komponisten an die zeitgenössische Musik heranführte. Zu jener Zeit wurde die musikalische Nachkriegsavantgarde sonst an keinem der Schweizer Berufskonservatorien unterrichtet. ${ }^{3}$

Die bei Boulez erlernte strukturelle Strenge und das serielle Regelwerk fesselten Holliger allerdings nur für kurze Zeit. Musikalischer Ausdruck, Expression war ihm zu wichtig, als dass ihn die distanzierten und sich teilweise selbst generierenden Kompositionssysteme des französischen Serialisten befriedigt hätten. Holliger war auch immer - und ist es bis heute emphatischer Interpret, und zwar keineswegs nur auf Neue Musik beschränkt. Gerade in den 6oer- und 7oer-Jahren spielte er auf über hundert Schallplatten fast das gesamte Oboen-Repertoire ein und war einem breiteren Publikum vor allem als Virtuose bekannt, von dem man allenfalls munkelte, dass er als Komponist völlig >verrückte< Musik komponiere. ${ }^{4}$ Holligers Kompositionen aus jener Zeit zählen tatsächlich zum Experimentellsten, was damals komponiert wurde, und nehmen in ihrer Körperlichkeit teilweise Entwicklungen der heutigen Performance-Szene voraus. So zum Beispiel bei Cardiophonie für ein Blasinstrument und drei Magnettonbänder, von Holliger selbst an der Musikbiennale in Zagreb 1971 uraufgeführt. Der Interpret fixiert Kontaktmikrofone auf seinem Brustkorb und spielt mit und zu seinem eigenen, für das Publikum hörbaren Herzschlag; dabei wird das Herz selbst Teil der Komposition, insofern der Oboist dessen Rhythmus gemäß der Partitur durch Pressen beim Blasen verändern bzw. beschleunigen muss. Dieses Stück, das den Körper und dessen überlebenswichtigen Blutkreislauf zum >Musikinstrumentく macht, endet in einem fingierten Kollaps.

Der Interpret Holliger führt als Komponist die Tradition des Virtuosentums weiter, sind doch seine Partituren extrem schwierig zu spielen und stellen höchste technische Ansprüche an die Interpreten. Allerdings handelt es sich nicht um eine äußerliche Virtuosität, vielmehr interessiert Holliger die psycho-physische Energie, die aus solcher Schwierigkeit und Anstrengung heraus entsteht. Viele Kompositionen Holligers sind geprägt von oft sehr

3 Vgl. Robert Piencikowski: Ein pädagogisches Experiment. Neben Heinz Holliger studierten zum Beispiel auch die Schweizer Komponisten Hans-Ulrich Lehmann (1937-2013) und Pierre Mariétan ("1935) zu jener Zeit in Basel.

4 So äußerte sich zum Beispiel in den 197oer-Jahren am Lehrerseminar Biel mein Musiklehrer Armin Schütz, der in der Sekundarschule in Langenthal auch den Knaben Holliger unterrichtet hatte. 
langen Auslöschungs-, Abbau-, ja Sterbeprozessen. Der Tod sei sein »Markenzeichen «, sagte Holliger einmal selbstironisch. ${ }^{5}$

Ich habe gar nicht so viele Ideen in der Musik, ich mache immer das gleiche - vom ersten Streichquartett an: Das ist ein einziges Rallentando von über 35 Minuten, ein einziges Wegnehmen von Energie, von Klanggenerierungsenergie, von Ausdrucksenergie, Saitenspannung, von Bogendruck wie auch von körperlicher Energie. ${ }^{6}$

Auch in Beiseit und Schneewittchen tut Holliger erneut das Gleiche, allerdings nicht mehr so kontinuierlich wie beim ersten Streichquartett, sondern konterkariert von Gegenbewegungen und Maskierungen aller Art. ${ }^{7}$

\subsection{Die >verrückten $<$ Dichter}

Von Jugend an war die Literatur für Holliger fast ebenso wichtig wie die Musik. Insbesondere mit der deutschen Literatur ist er aufs Engste verbunden. ${ }^{8}$ Die von ihm vertonten Dichterinnen und Dichter weisen fast durchwegs sonderbare oder tragische Biografien auf: Sie nahmen sich das Leben (Paul Celan, Alexander Xaver Gwerder und wohl auch Georg Trakl) oder sie landeten in Nervenheilanstalten (Friedrich Hölderlin, Nikolaus Lenau, Robert Schumann, Louis Soutter und Robert Walser). Diese >verrückten< Lebensläufe ziehen Holliger magisch an, und er verarbeitet in vielen seiner Werke die Lebenskontexte dieser Dichter und Künstler. Beim Scardanelli-Zyklus hat Holliger dies erstmals erprobt, indem er nicht nur die späten Gedichte, sondern auch Hölderlins Leben und vor allem seine Zeit im Tübinger Turm in Musik fasste. Mit 36 Jahren begann Holliger mit der Komposition; die Arbeit daran dauerte 16 Jahre und führte zu seinem bisher umfangreichsten und längsten Werk. Ebenfalls 36 Jahre alt war Hölderlin, als er gegen seinen Willen am 11. September 1806 ins Tübinger Klinikum von Johann Hermann Heinrich Ferdinand von Autenrieth eingeliefert wurde. Von

5 Olshausen: Ein Stückchen weit.

6 Holliger/Zimmermann: »Ich hoffe, ich habe nie in meinem Leben etwas sver-tont««, S. 23.

7 Interessant ist, dass Holliger bei seiner dissoziativ konzipierten Oper Lunea nach Nikolaus Lenau (2017) auf ein Libretto von Händl Klaus ursprünglich wirklich etwas anderes machen und einen ätherisch hellen, quasi >schönen< Schluss mit Äolsharfen gestalten wollte. Nach langem Überlegen und Experimentieren wurde ihm das aber doch zu pathetisch und oberflächlich, und er machte erneut das >Gleiche $:$ Wie durch einen extremen Filter hindurch ist ein sechsstimmiger Choral der Blechbläser zu vernehmen, eine Musik wie im Schatten einer Kerze, senza fine - wie bei den Schlüssen von Beiseit und Schneewittchen. Vgl. Brotbeck: »Der Mensch ist ein Strandläufer am Meer der Ewigkeit«.

8 Vgl. Olshausen: Heinz Holliger und die Literatur. 
Autenrieth war ein >fortschrittlicher Psychiater, denn er war überzeugt, dass psychisch Kranke nicht nur verwahrt werden sollten, sondern sich auch heilen ließen. Er diagnostizierte bei Geisteskranken zu großen >Eigensinn<, den es zu brechen gelte. Dies führte zu teilweise lebensgefährlichen Therapien. So verätzte er die Haut seiner Patienten, um Entzündungen auszulösen, oder er zwang sie mit Zaummasken zum Schweigen. Auch Hölderlin wurde solchen Therapien unterzogen, die aber im Frühjahr 1807 ein Ende nahmen: Hölderlin wurde als unheilbar geisteskrank entlassen und in den Haushalt des Tischlermeisters Ernst Zimmer aufgenommen. Hier lebte er 36 Jahre lang in einem Turmzimmer und schrieb - oft für eine Pfeife Tabak - wieder Gedichte. Er versah diese mit falschen Datierungen der Vergangenheit und Zukunft und unterzeichnete sie häufig mit Scardanelli.

Der Schweizer Germanist Bernhard Böschenstein, der in den späten 195oerJahren im Gymnasium Burgdorf Heinz Holliger während einer Stellvertretung unterrichtet hatte, war 1965 der erste, der die hohe Qualität und Modernität der späten Gedichte von Hölderlin erkannte und sie in den Kontext von Paul Celans Lyrik stellte. ${ }^{9}$ Bis dahin wurden diese >ich-verneinenden< Gedichte nur unter pathologischen Gesichtspunkten betrachtet.

Im Scardanelli-Zyklus werden von Autenrieths Zaummasken gewissermaßen in Musik übersetzt: Exerzitien, leer drehende Kanons, Klänge an der Grenze der Wahrnehmbarkeit; die Sängerinnen und Sänger müssen während des Einatmens jubilierende Akkorde singen; der Flötist muss mit leerer Lunge blasen. Normalerweise gibt der Komponist an, wo geatmet werden darf; im Scardanelli-Zyklus ist "nicht einatmen « eine neue und häufig auftretende Spielanweisung.

Der Abschluss des Zyklus und die Auseinandersetzung mit Beiseit überschneiden sich: 1991, ein Jahr nachdem er Beiseit beendet hatte, schloss Holliger die sechzehn Jahre dauernde Arbeit am Scardanelli-Zyklus mit dem Ostinato funebre ab. Dieser Satz korrespondiert mit der ersten Hälfte von Hölderlins Leben. Während die meisten Teile des Scardanelli-Zyklus wie erkaltet wirken, wie Totenmasken, herrscht in diesem Stück das Leben; es gibt Aufbrüche, Kontraste und gespenstische Naturstimmungen. Die unmittelbar vorangegangene Erfahrung mit Robert Walser scheint hier ihre Spuren hinterlassen zu haben. Ostinato funebre wie Beiseit kündigen eine neue, subjektivere, emotionalere Welt an, die Holligers Schaffen seither dominiert. Und während man in den Vokalteilen des Scardanelli-Zyklus die Worte kaum versteht, weil sie in der Musik gleichsam zerrieben und aufgelöst werden, ist in Beiseit alles so gesetzt, dass der Text nachvollzogen werden kann.

9 Vgl. Böschenstein: Hölderlins späteste Gedichte. 


\subsection{Autobiografische Identifikation}

Die Beschäftigung mit Walser hat bei Heinz Holliger ein autobiografisches Moment, das sich durch verblüffende Parallelen erklärt. Wie Robert Walser mit Karl Walser hatte auch Heinz Holliger einen nur wenig älteren Bruder, den Schauspieler und Regisseur Erich Holliger (1938-2010). Er war für Heinz Holliger gerade in der Jugendzeit und während der ersten künstlerischen Arbeiten von zentraler Bedeutung. Heinz Holliger war eine Frühbegabung, ähnlich wie Robert Walser - mindestens im Vergleich zu Schweizer Schriftstellern wie Conrad Ferdinand Meyer oder Gottfried Keller. Schon als Jugendlicher trat er solistisch auf - treffenderweise auch mit dem ersten Schweizer Walser-Vertoner Wilhelm Arbenz. ${ }^{10}$ Holliger wurde verschiedene Male von der Familie Arbenz eingeladen, aber über Robert Walser habe man sich da nie unterhalten. ${ }^{11}$ Auch Walsers Unverfrorenheit findet sich beim jungen Holliger. So schrieb er mit sechzehn Jahren im jugendlichen Übermut dem Basler Mäzen, Dirigenten und Verwaltungsratspräsidenten von HoffmannLa Roche Paul Sacher, um die Adresse von Benjamin Britten zu erbitten und zu empfehlen, neue Oboenkonzerte in Auftrag zu geben. ${ }^{12}$ Ein großer Unterschied zwischen Walser und Holliger liegt im Erfolg, der Holliger beschieden, Walser aber zu seinen Lebzeiten weitgehend verwehrt geblieben war.

Holliger hatte das Glück, bei den besten Lehrern seiner Zeit studieren zu können. Oboe lernte er bei Emile Castagnaud in Bern und Pierre Pierlot in Paris, Klavier bei Sava Savoff in Bern und Yvonne Lefébure in Paris. Für Komposition, Theorie und die allgemeine musikalische Bildung ist Sándor Veress wichtig, der wie ein künstlerischer Vater den hochbegabten Knaben am Berner Konservatorium in alle musikalischen Disziplinen einführte, ihm als Universalgelehrter die gesamte Kulturgeschichte eröffnete und auch interpretatorisch wichtige Impulse gab, indem er ihn lehrte, Werkanalyse und Interpretation direkt zu verbinden. Diese tiefen Einsichten in die kompositorische

101956 spielt Holliger in der Kirche Biel-Mett unter Arbenz das Oboenkonzert von Domenico Cimarosa, dazu noch Händels g-Moll-Sonate. Das Honorar für den siebzehnjährigen Solisten betrug fünfzig Franken; Holligers Mutter fand die Fünfzigernote später nach dem Waschen in der Hose ihres Sohnes. E-Mail von Barbara Golan (Agentin von Heinz Holliger) an Roman Brotbeck vom 15.11.2016.

11 Ebd.

12 Bachmann-Geiser: Heinz, S. 162f. Als Holliger vier Jahre später 1959 als Zwanzigjähriger den Genfer Musikwettbewerb gewann, lud ihn Sacher ein und holte den Brief aus der Schublade. Holliger durfte nun tatsächlich bestimmen, an welche Komponisten Sacher Aufträge vergeben sollte. 
Struktur und den musikalischen Gehalt eines Werkes zeichnen bis heute die zahlreichen Interpretationen von Holliger aus. Einige Unterrichtsstunden bei Veress - zum Beispiel jene zu den Romanzen op. 95 für Oboe und Klavier von Robert Schumann - haben sich Holliger so tief eingeprägt, dass sie seine Interpretation dieses Werks bis heute beeinflussen. ${ }^{13}$ Veress wird für den Komponisten Holliger im Verlauf seines Lebens zunehmend wichtiger; gerade Beiseit enthält viele Reminiszenzen an seinen ersten Kompositionslehrer.

Es gibt im Falle von Beiseit - wie schon beim Scardanelli-Zyklus - eine weitere biografische Auffälligkeit: War Holliger bei seiner ersten Beschäftigung mit Hölderlin 36-jährig und damit gleich alt wie jener zur Zeit seiner Einweisung ins Tübinger Klinikum, beginnt er die Beiseit-Komposition im Alter von $5^{1}$ Jahren, exakt jenem Alter also, in dem sich Robert Walser Anfang 1929 auf Drängen seiner Schwester Lisa in die Heilanstalt Waldau bei Bern begibt.

Die Werkidee zu Beiseit bilden Jahre zuvor Pläne, etwas `Schweizerisches< zu vertonen. Walser steht damals noch nicht exklusiv im Zentrum der Überlegungen. Vielmehr denkt Holliger an »Schweizer Lieder für Countertenor, Akkordeon, Klarinette « ${ }^{14}$ mit Texten von Heinrich Leuthold, Conrad Ferdinand Meyer, Robert Walser und Adolf Wölfli - alles Schriftsteller, die im Irrenhaus gelandet waren. Zu letzterem hatte Holliger schon »1972 ein Riesen-WölfliProjekt entworfen «.15

Ich wollte auch ein $\mathrm{CH}$-Quartett machen, auf einem riesigen Faß, das in Hunderte von Instrumenten verwandelt wird durch Einfügen von Mundstücken, von Trommelfellen, von Saiten usw. Das wäre vor allem auf Texte von Wölfli gewesen, aber ich habe es damals leider nicht gemacht. Langsam hat sich vielleicht dieses kämpferische Schweizerische sublimiert und ist immer feiner geworden, bis es bei Walser landen musste. ${ }^{16}$

Die Besetzung für Kontratenor, Klarinette, Akkordeon und Kontrabass, ${ }^{17}$ also für die Standardinstrumente der Schweizer Volksmusik, steht für Holliger damals aber schon fest. In der Schweizer Volksmusik liefern die Schwyzerörgeli-Spieler

13 Trotz seiner konservativen Prägung muss Veress heute als einer der wichtigsten Kompositionslehrer des 2o. Jahrhunderts bezeichnet werden, denn der Bartók-Schüler hat nicht nur in Bern viele Schweizer Komponisten ausgebildet, sondern zuvor in Ungarn auch György Ligeti und György Kurtág.

14 Holliger/Zimmermann: IIch hoffe, ich habe nie in meinem Leben etwas sver-tont «, S. 18.

15 Ebd., S. 19.

16 Ebd. Der Plan erinnert an die Fantasien des Knaben Heinz, der Oboe lernen wollte und sich - nachdem ihm jemand von den Mundstücken der Oboe erzählt hatte - eine Apparatur mit einem Bunsenbrenner und Schläuchen vorstellte. Holliger erzählt diese Geschichte in Olshausen: Ein Stückchen weit.

$17 »[\mathrm{I}] \mathrm{ch}$ weiß nicht mehr, ob ich Kontrabass auch schon dabei hatte ${ }^{\prime}$. Holliger/Zimmermann: »Ich hoffe, ich habe nie in meinem Leben etwas sver-tont««, S. 18. 
oft ziemlich mechanisch und >nebenbei< ihre präformierten Formeln zum Tanzgeschäft ab. Holliger gibt dem Akkordeon eine ähnliche Rolle: Es steht im Zyklus quasi im >Beiseit<, kommt aus der Ferne, keucht mit leerem Balg vor sich hin, wird auch weniger in die verwendeten Formzitate einbezogen.

Den Kontratenor wählte Holliger aus drei Gründen: wegen seiner Nähe zu falsettierenden Männerstimmen im alpenländischen Jodel, wegen des adoleszenten, geschlechtlich noch ambivalenten Moments in der Stimme des Kontratenors, das für Holliger mit Walser direkt korrespondiert, zumal in diesen frühen Gedichten Erotisches absent ist. Schließlich war Holliger an der Spaltung zwischen Sing- und Sprechstimme interessiert. Der Kontratenor hat meist eine relativ tiefe Sprechstimme in Baritonlage. Die beiden Stimmtypen des Kontratenors scheinen verschiedenen Persönlichkeiten anzugehören. ${ }^{18}$ Am eindrücklichsten kommt diese schizophrene Disposition beim neunten Gesang Angst zum Vorschein, wo die beiden Stimmtypen miteinander dialogisieren und sich die Identität des lyrischen Ichs aufspaltet.

Dieses Interesse für die gespaltene Stimme zeigt, dass Holliger in Beiseit Walsers frühe Gedichte nicht in ihrer Entstehungszeit belassen, sondern sie in Bezug zum gesamten CEuvre von Robert Walser stellen möchte: »Mir war die Idee eines Lebenslaufs ganz wichtig. ${ }^{19}$ Er interessiert sich deshalb besonders für jene Momente in den Gedichten, die prospektiv in die Zukunft weisen.

\subsection{Formmasken}

Walsers Lebenslauf mit seinen weiten Ausschlägen nach Zürich, Basel, Würzburg, Berlin in jungen Jahren, der Rückkehr nach Biel, dem »Prosastückligeschäft « ${ }^{20}$ (BA 2, 358) sowie dem >Bleistiftgebiet « in Bern und der letztlich doch freiwilligen Einweisung in die Psychiatrische Klinik Waldau bis hin zum vollständigen Verstummen nach der Entmündigung und Zwangsüberweisung nach Herisau im Kanton Appenzell Ausserrhoden stimmt mit Holligers Rallentando-Formen perfekt überein. Endpunkt ist der letzte Vers von Im Mondschein: »lag lang noch wach« (SW 13, 20). Holliger bezieht dies

18 Der Kontratenor (auch Altus oder Countertenor) kommt in den Walser-Vertonungen relativ oft und prominent vor, vgl. Aschenbrödel von Martin Derungs (Kap. 8.5.2) und Johannes Fritsch (Kap. 13.3) sowie Es kommt mich ... von Mathias Steinauer (Kap. 11.15.2). Zu erwähnen wäre auch die Wölfli-Kantata von Georges Aperghis (Kap. 7.6).

19 Holliger/Zimmermann: »Ich hoffe, ich habe nie in meinem Leben etwas sver-tont«", S. 21.

20 Die Bezeichnung »Prosastückligeschäft« ist in der Walser-Literatur schon fast zu einem geflügelten Wort geworden, obwohl sie Walser selbst nur ein einziges Mal, 1928 in einem Brief an Frieda Mermet, verwendet. Dank an Gelgia Caviezel für diese Recherche. 
auf die Jahre in der Psychiatrie: »[D]iese Wachheit, das ist bei Walser immerhin die Zeit von 1929 bis $1956 . \ll^{21}$

Dem Rallentando setzt Holliger ein anderes Formprinzip entgegen, das die abnehmende >Klanggenerierungsenergie $<$ bremst, überdeckt und maskiert: ein Spiel mit wechselnden Formen und Genres, das Holliger schon in Albchehr für die Oberwalliser Spillit ausgiebig erprobte. »Ich wollte in Beiseit [...] nicht so eine Einbahnstraße komponieren wie im Streichquartett, das einfach stur in eine Richtung bis zum Exitus geht.«22

Beim Scardanelli-Zyklus erscheint immer nur jene Maske, die man weder abnehmen noch wechseln kann: die Totenmaske. Im Beiseit-Zyklus spart sich Holliger diese Maske für das Ende auf. Zuvor spielt er mit den vielen Maskierungen bei Walser, indem er für jedes Lied eine eigene Stilmaske wählt. Es ist ein ähnliches Verfahren, wie es Alban Berg in der Oper Wozzeck gewählt hat, indem er jede Szene der Oper in eine bestimmte Form setzt, die inhaltlich mit der Szene korrespondiert. Auch bei Holliger stehen Form und Text in engstem Bezug. Wie die folgende Kurzbeschreibung der zwölf Lieder zeigt, ist Holliger bei der Wahl der Formmasken selbst in seine Jugendzeit zurückgekehrt; vor allem der Einfluss von Sándor Veress ist unüberhörbar, aber auch Alban Berg und andere vermeint man zu hören.

\section{Beiseit (SW 13, 22) für Kontratenor, Akkordeon und Kontrabass} Form: Passacaglia

Akkordeon in höchster und Kontrabass in tiefster Lage sind spiegelsymmetrisch geführt, die Singstimme am Anfang ruhig schreitend, später mit immer unsichererem Gang. Die Melodie ist ikonografisch den letzten Schritten von Walser im Schnee nachgebildet (vgl. Kap. 10.7); dabei hat die Zahl 6, die anfänglich mit sechs Doppelschritten exponiert wird, im Lied strukturelle Bedeutung. Bis zur Mitte des Liedes verwendet Holliger eine symmetrisch konstruierte Zwölftonreihe.

\section{Schnee (I) (SW 13, 15) für das ganze Ensemble}

Form: Proportionskanon

Ein dreistimmiger Kanon: Die Klarinette spielt in anderthalb-, der Kontrabass in zweieinhalbfacher Vergrößerung die anfänglich in kleinem Ambitus geführte Melodie in der Singstimme. Alle Stimmen befinden sich in der gleichen Lage, beginnend beim >Todeston $d$; »das $[\ldots]$ ist ganz eng verwandt

\footnotetext{
21 Ebd., S. 23.

22 Ebd., S. 26.
} 
mit den Kanons über >Herbstく im Scardanelli-Zyklus, die alle über dieses $d$ komponiert sind.« ${ }^{23}$ Beim Wort »Tränen« bricht die Singstimme in ein überspanntes Melisma aus. Klarinette und Kontrabass erreichen mit ihren langsamer voranschreitenden Kanonstimmen diesen Ausbruch nicht mehr. Das Akkordeon ist in den Kanon nicht eingebunden und spielt nur die Bandbreite des sich ausweitenden Ambitus.

III Bangen (SW 13, 26) für Kontratenor, Akkordeon und Kontrabass Form: Valse lente

Stark chromatischer >fin de siècle<-Walzer mit mehrfach leittöniger Harmonik, »der fast noch etwas vom Fin de siècle hat, das man auf den Bildern Karl Walsers sieht: etwas fast Süßes, Melancholisches, Weiches, Elegantes.«24 Kontrabass und Akkordeon erklingen hier erstmals dolce und in ihrer natürlichen Lage.

IV Wie immer (SW 13, 14) für Kontratenor und Kontrabass Form: Melodram

Hier brechen die Form-Masken erstmals auf, »da geht es schon ganz ins Existentielle. Denn der Kontrabass wird wie ein Körper quasi gefoltert, wird manipuliert mit Werkzeugen, er schreit, ächzt, seufzt [...], es ist eine völlig zerbrochene Musik. «25 Der Kontratenor tritt hier als Bariton auf und spricht in tiefer Lage; nur wenige Wörter werden gesungen, was zu einer schizophrenen Spaltung der Stimme führt. Für Holliger entstehen hier Bezüge $\mathrm{zu} »$ Cardiophonie: der gefolterte Körper, der zu Musik wird. $\ll^{26}$

\section{Trug (SW 13, 28) für Kontratenor, Klarinette (und Kontrabass)}

Form: Bicinium

Gesangsstimme und Klarinette greifen so eng ineinander, dass keine Hauptstimme auszumachen ist. Gewisse Reizwörter wie »drehen« und »weinen« sind madrigalistisch herausgestellt. Am Schluss führt der Kontrabass das Bicinium der verstummten Singstimme zu Ende. Für Holliger ist dieser Satz »ein äußerlich harmloses Bicinium [...], das noch ein bisschen anklingt an ein Genre-Stück. Aber es ist schon das Taumeln drin, [...] die Welt fängt an zu schwanken.«"27

23 Ebd., S. 24. Vgl. auch die Analyse des Liedes Schnee (I) von Lanz: »... die weißverschneite Welt macht mich schwach«, S. 6o-66.

24 Holliger/Zimmermann: »Ich hoffe, ich habe nie in meinem Leben etwas sver-tont «, S. 24.

25 Ebd.

26 Ebd.

27 Ebd. 
VI Zu philosophisch (SW 13, 23) für das ganze Ensemble (Bassklarinette) Form: Perpetuum mobile I

Das Wort »Redeflechter « im Gedicht scheint die Vertonung beeinflusst zu haben: ein schnelles und dichtes Flimmern verschiedener in sich drehender Motive, »die Aufhebung des normalen Zeitgefühls und die Aufhebung der Körperlichkeit, auch beim Instrument, der Bassklarinette: >On fait marcher les doigts! <, wie Aurèle Nicolet zu sagen pflegte $\ll_{.}^{28}$ Die Singstimme verläuft in wilden Sprungbewegungen, »schon ein bisschen sprechgesangartig, wie ein Recitativo accompagnato. ${ }^{29}$ Am Schluss des Liedes wird dieser Spuk von der Bassklarinette wie weggeblasen.

VII Abend (I) (SW 13, 8) für das ganze Ensemble Form: Litanei

Die mikrointervallisch eng geführten Stimmen umspielen den Ton es. Die dadurch entstehende gepresste Wirkung erinnert an die Teile Sommer II und Engführung des Scardanlli-Zyklus. »Der Gesang [...] wird nun völlig gedehnt in riesenlange Vokalisen, unendlich viele Verzierungen - wie Gregorianik oder wie in den ungarischen Siratók. [...] Man weiß überhaupt nicht mehr, wo man ist, und das ist ja genau der Inhalt des Gedichtes. ${ }^{30}$

VIII Weiter (SW 13, 18) für das ganze Ensemble Form: Scherzo

Dieses Lied ist geprägt von komplexen rhythmischen Überlagerungen und Verschiebungen; zweimal - synchron zum Wort »Rauch « - gelangt die Bewegung zum Stillstand. Anschließend steigen Singstimme und alle Instrumente langsam und kontinuierlich nach oben. Dieses Lied unterbricht den Sog abnehmender Klangenergie, »ein bisschen eine >Hommage à Veress $<$, in ungarischer Rhythmik. Das ist vielleicht der übermütige Simon Tanner aus den Geschwistern Tanner, es zeigt das Jungenhafte von Walser. Plötzlich bleibt es auf einem Ton stehen, dann geht es wieder weiter. ${ }^{31}$

IX Angst (I) (SW 13, 15f.) für das ganze Ensemble (Bassklarinette)

Form: Dialog

Die im vierten Lied erstmals angedeutete Aufspaltung der Kontratenorstimme in eine Baritonsprechstimme und in eine Altsingstimme isthier als wilderDialog

\begin{tabular}{ll}
\hline 28 & Ebd., S. 25. \\
29 & Ebd. \\
30 & Ebd., S. 26. \\
31 & Ebd.
\end{tabular}


zwischen den beiden Stimmtypen gestaltet. In der Instrumentalbegleitung wird das Schauerliche dieser gespaltenen Persönlichkeit mit zahlreichen ungewöhnlichen Effekten betont.

$\mathrm{X}$ Und ging (SW 13, 27) für Kontratenor, Klarinette und Akkordeon Form: Tema con 4 variazioni

Die Variationen sind kontrastreich und programmatisch angelegt. Wenn von der »Majestät« die Rede ist, wird zum Beispiel eine französische Ouvertüre imitiert. Vergangenes - Mozart und Schumann - flackert auf. »Das ist wie eine Rückschau, die aber nicht gelingen will.«32

XI Drückendes Licht (SW 13, 25) für das ganze Ensemble Form: Lied

Eine dreiteilige Liedform mit stark kontrastierendem Mittelteil. Die Singstimme entfaltet sich hier sehr frei, ihre langen Melismen wirken zum Teil fast opernhaft. In den zerdehnten Tönen der Außenteile wird die tödliche Ruhe und Länge des letzten Liedes vorbereitet.

In »Drückendes Licht« löse ich gleichzeitig auch die Schwerkraft der Tonalität auf. Da ist alles mit reinen, untemperierten Obertönen geschrieben, aber man hört noch kleine Anklänge an klassische Musik. Die Musik wird schon völlig schattenhaft, und es endet hier mit einem Anklang an einen Walzer-Rhythmus [...]: Plötzlich ist das wieder wie »Valse lente«. [...] Das ist eigentlich der Schluss des Zyklus. Aber es könnte natürlich überhaupt nicht hier enden. ${ }^{33}$

XII Im Mondschein (SW 13, 19f.) für das ganze Ensemble (Bassklarinette), Akkordeon im Bühnenhintergrund, aber sichtbar

Form: Perpetuum mobile II

Das Perpetuum mobile II ist sehr viel breiter angelegt als das erste Perpetuum (Lied VI). Dieses Mal wird die unentwegte Bewegung nicht ausgeblendet, sondern so stark verlangsamt, dass die Singstimme an ihre atemtechnischen Grenzen stößt. Bei diesem letzten Lied verengt sich das Maskenspiel - wie beim Scardanelli-Zyklus - auf die eine Maske, die kein Spiel mehr ist: die Totenmaske.

\begin{tabular}{ll}
\hline 32 & Ebd. \\
33 & Ebd., S. 27.
\end{tabular}




\section{5 >Eichendorff'sche Mondnacht}

Das letzte Lied aus dem Zyklus Beiseit ist ein halbes Jahr nach den anderen elf Liedern entstanden. Es ist das einzige, in dem ein anderer Komponist deutlich zitiert wird. Zwar gibt es auch in den anderen Liedern Anspielungen etwa auf Mozart, Schubert, Schumann und Berg, aber nie als präzises Zitat, mehr der Erinnerung nach. Im letzten Lied bezieht sich Holliger nun aber explizit auf die Mondnacht aus Schumanns Liederkreis op. 39. Walser selbst gab Holliger dazu die Idee, denn er schreibt im Prosastück Würzburg:

Mitternacht war es, und statt des Schlafes [...] genoß ich nun den Anblick der schönsten Mondnacht, die wie eine Eichendorffsche Mondnacht alle ihre unnennbare Schönheit [...] niederwarf. Eine leise Handharfe ertönte[.] $($ BA 15,40$)$

Wegen dieser Stelle in Würzburg verlangt Holliger, dass das Akkordeon in diesem letzten Lied als »leise Handharfe « aus dem Hintergrund ertönen muss.

Für Holliger ist auch der Schluss von Schumanns Vertonung von essentieller Bedeutung. In der letzten Strophe von Eichendorffs Gedicht heisst es: »Und meine Seele spannte / weit ihre Flügel aus, / flog durch die stillen Lande, / als flöge sie nach Haus. « Dieses >nach Haus< wird von Schumann in einmaliger Weise enttäuscht, indem er anstelle der lang vorbereiteten Tonika eine Dissonanz, nämlich den Dominantsextakkord auf der ersten Stufe, also der Tonikastufe, platziert. Das >nach Haus < verweist in eine fremde Welt und wird zum Zeichen für die nicht mögliche Rückkehr. Dieses Vorgehen wirkt umso schockartiger, als sich Schumann zuvor an die Strophenform gehalten hat.

Dieses Verfahren, mit einer strophischen Anlage eine Erwartung aufzubauen und diese dann umso deutlicher zu enttäuschen, übernimmt Holliger bei den längeren Gedichten im Beiseit-Zyklus. Mit der deutlichen Anlehnung an Schumann ergibt sich im letzten Stück die komplexeste Strophenform. Holliger lässt die Schumannreminiszenz mit dem eine Quarte durchschreitenden aufsteigenden Motiv von Strophe zu Strophe deutlicher werden, bis es bei »noch lange hört' ich es singen « für einen kurzen Moment nicht mehr zu überhören ist.

Nach dieser Annäherung an Schumann folgt in Holligers Vertonung der Schock, hier allerdings nicht mit einer unerwarteten Modulation, sondern mit einer gleichsam außer Rand und Band geratenen Ausdeutung des Wortes »singen«, das sich in einem nicht enden wollenden Melisma über zwei Systeme hinzieht. 

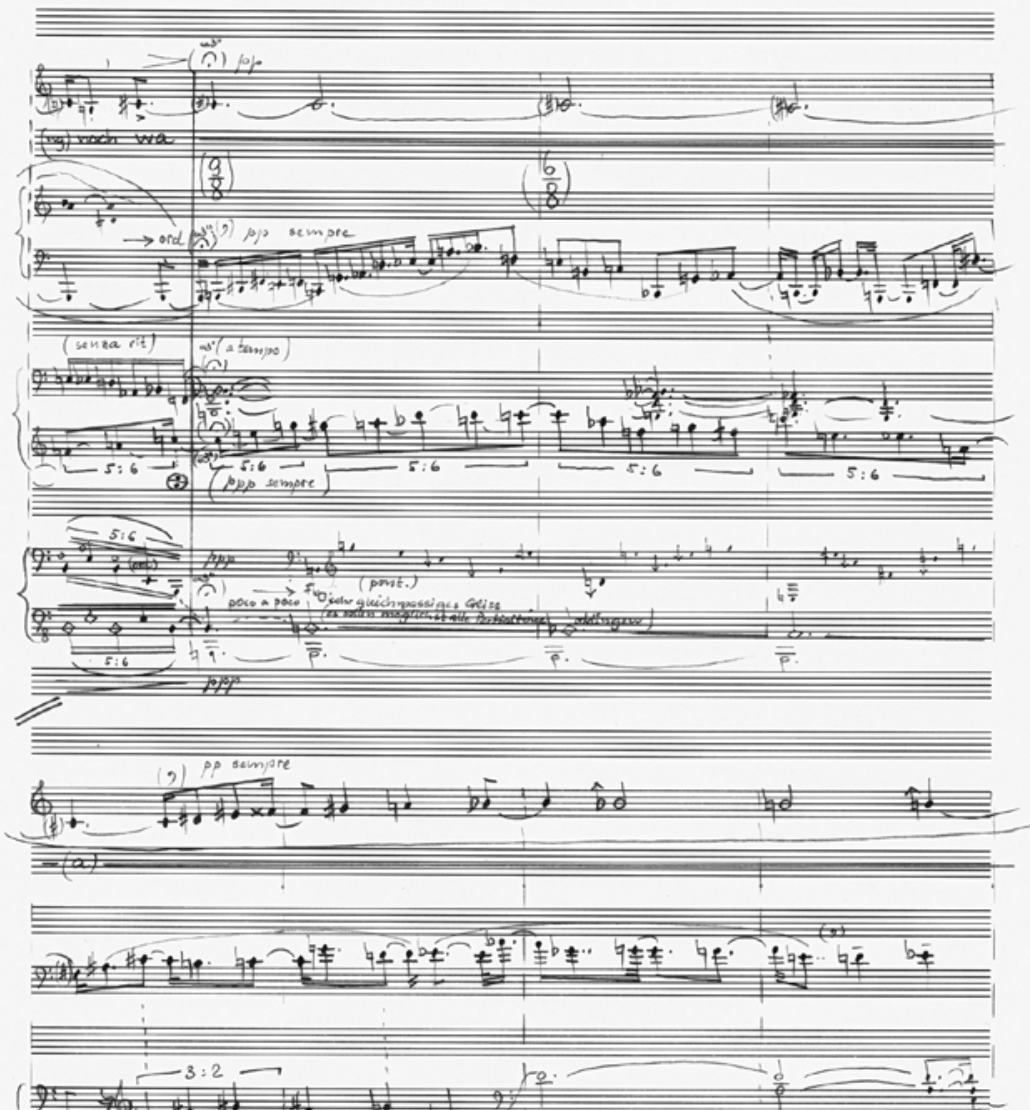

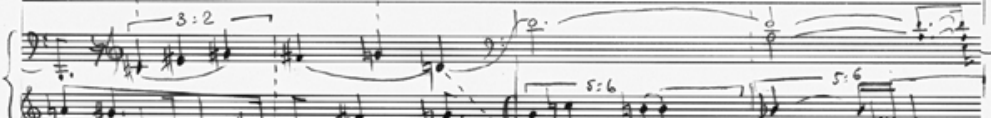

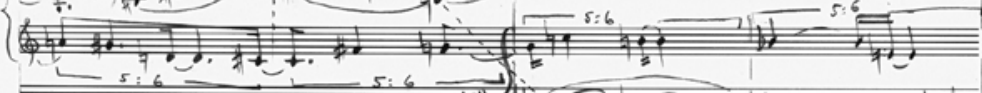

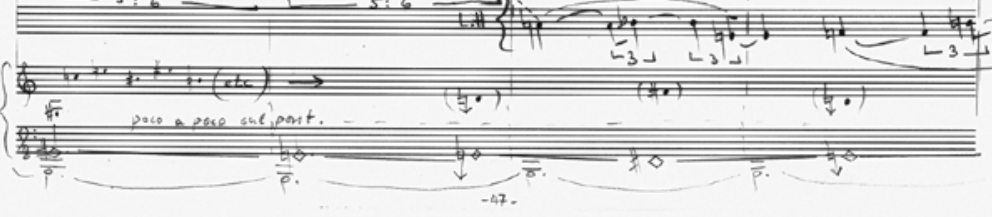

Abb. 21 Heinz Holliger: Im Mondschein, S. 47f. (Schluss) 


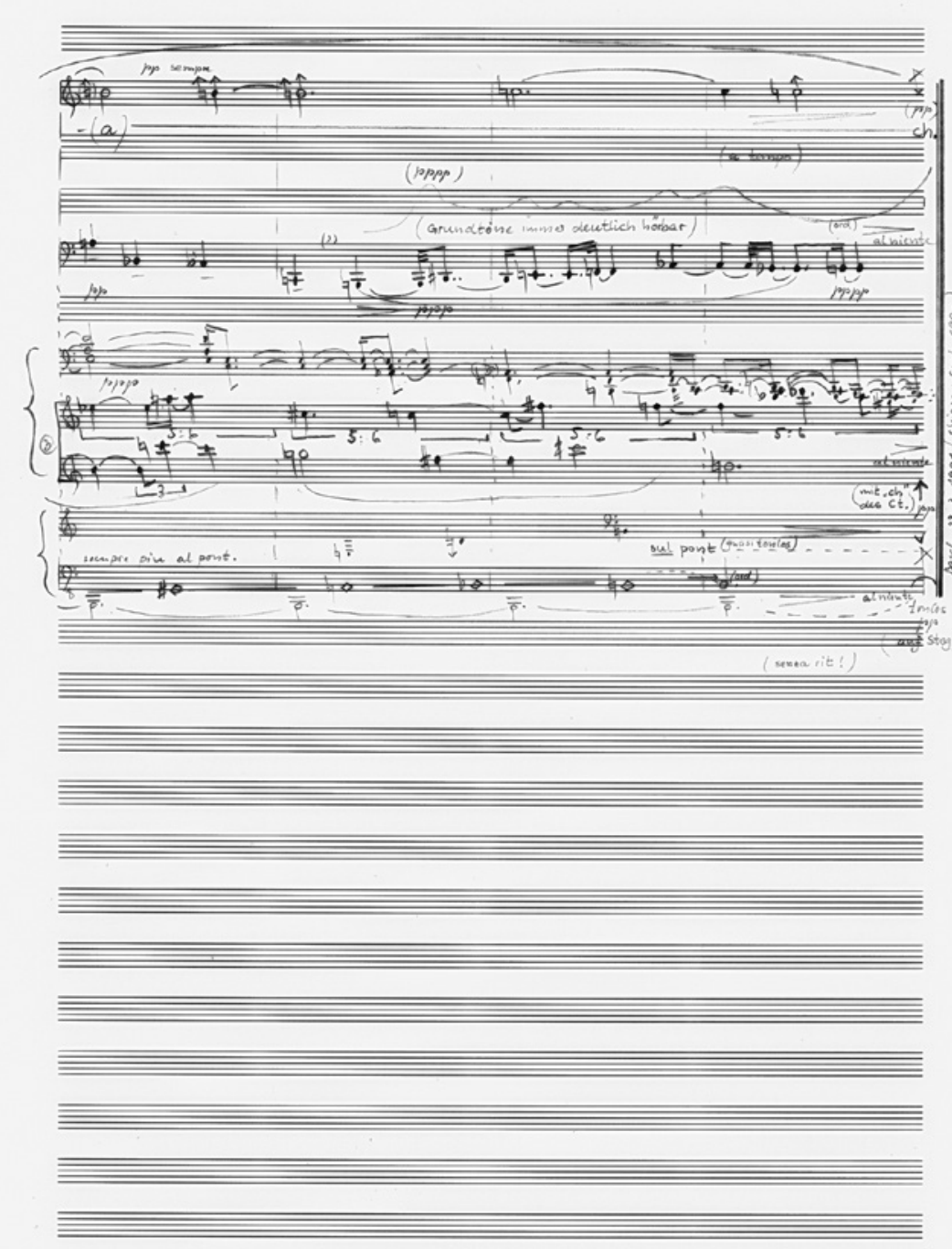

$-48-$ 
Diese Wirkung wird noch gesteigert beim nachfolgenden »lag lang noch wach«, indem die Musik auf »wach « mit immer längeren Tönen quasi stehenbleibt (vgl. Abb. 21).

Über dem Orgelpunkt auf dem Ton $H$, mit dem das Stück begonnen hat und der die >Signatur von Heinz Holliger darstellt, wird von cis $^{1}$ aus der Oktavraum durchschritten. Die Notenwerte werden kontinuierlich länger, die Tonschritte ebenso kontinuierlich kleiner. Auch hier erklingt nach der Atempause eine >Zwölftonreihe<, allerdings ist es keine >richtige< mit Halbtönen wie beim ersten Lied Beiseit (vgl. Kap. 10.7): Die Skala steigt zuerst in drei (enharmonisch richtig notierten) Ganztönen, dann in drei Halbtönen, drei Vierteltönen und schließlich in drei Achteltönen nach oben. Der die Oktave einlösende und die Skala >erlösende Oktavton cis $^{2}$ wird jedoch nicht erreicht. Auch diese letzte Skala leitet sich aus Schumanns Mondnacht ab, dessen aufsteigendes Skalenmotiv hier gleichsam bis zum konsequenten Ende gedacht wird.

Am Ende, auf das »-ch « - wie wenn man eine Kerze ausbläst - bricht das Stück $\mathrm{ab}$, wie mit der Schere weggeschnitten. Es ist eigentlich kein Schluss, die Musik könnte jahrelang weitergehen, immer enger. ${ }^{34}$

Der Schluss von Schumanns Mondnacht steht für die nicht mögliche Rückkehr, jener von Holligers Im Mondschein für das Nicht-Erreichen-Können der »geheimnisvolle[n], nie erklärbare[n] Schwelle, die zu überschreiten keinem Lebenden je gegeben ist «. ${ }^{35}$

Die Zahl Sechs, die im ersten Lied Beiseit als einfache Umsetzung von Walsers letzten Schritten im Schnee erscheint, verwandelt sich in Im Mondschein zu einem alle Parameter bestimmenden Zahlencode. Die Diastematik des Schrittmotivs von Beiseit - ein verlangsamtes Wandermotiv - ist nun zu einer leicht gekrümmten Geraden erstarrt: Die letzten Spuren des lebenden Walser im ersten Lied sind am Schluss des letzten Liedes, an dem Heinz Holliger länger gearbeitet hat als an allen anderen Liedern zusammen, zur abstrakten Todeslinie geworden.

Es war mir ganz wichtig, dass ich plötzlich diese Schritte ganz von außen her durch die Reflexion reinzwinge, bis genau der Punkt kommt, an dem klar ist, entweder muss ich hier aufhören, oder dann erst viel viel später.

Ganz ähnlich bei Schneewittchen, wo es heißt: »Kommt alle mit herein « - ins Unterbewusste. Es hat keinen Schluss. ${ }^{36}$

34 Ebd., S. 3 o.

35 Gemäß Mail von Barbara Golan an Roman Brotbeck vom 20.03.2021. Holligers Kommentar bezieht sich auf Franz Schubert.

36 Holliger/Zimmermann: »Ich hoffe, ich habe nie in meinem Leben etwas sver-tont«", S. 3 o. 


\subsection{Schneewittchen}

Ende des letzten Jahrhunderts beschäftigten sich gleich drei führende, der Avantgarde zuzuordnende Komponisten mit Märchenopern: Johannes Fritsch mit der Aschenbrödel-Version von Robert Walser (noch nicht aufgeführt; vgl. Kapitel 13.3), Helmut Lachenmann mit Hans Christian Andersens Das Mädchen mit den Schwefelhölzern (UA 1997 in Hamburg) und Heinz Holliger mit dem Dramolett Schneewittchen von Robert Walser. Martin Zenck hat die beiden Opern von Holliger und Lachenmann verglichen und vermutet, dass die fast gleichzeitige Auseinandersetzung mit einem Märchenstoff kein Zufall sei. ${ }^{37}$ Mit mehr zeitlichem Abstand zeigt sich allerdings, dass die Opern von Lachenmann und Holliger letztlich wenig miteinander zu tun haben. Zwar greifen beide Komponisten biografisch stark in ihre Jugendzeit zurück: Helmut Lachenmann setzt sich mit der RAF-Terroristin Gudrun Ensslin auseinander, mit der er als Kind im Sandkasten spielte, und Heinz Holliger kehrt teilweise in die Fantasiewelt seiner Jugend zurück und stellt einen direkten Bezug zum Weihnachtstag 1956 her, an dem Holliger in Langenthal der Mutter sein Lied Schmetterling nach Morgenstern schenkte, während Robert Walser bei Herisau von Kindern tot im Schnee gefunden wurde.

Damit sind die Parallelen weitgehend erschöpft, denn Lachenmann führt mit der Überschreibung von Andersens Mädchen mit der Brände stiftenden Gudrun Ensslin letztlich das große Projekt politischen Engagements weiter. In musikalischer Hinsicht ist das Mädchen die konsequenteste Umsetzung von Lachenmanns >Musique concrète instrumentale $<$ Ein ganzes Orchester und alle Stimmen erzeugen die hochdifferenzierte Geräuschkomposition. Im Unterschied zu Heinz Holliger zeigt Lachenmann ein tiefes Misstrauen gegenüber der Gesangsstimme und ihrem überhöhenden und emphatischen Charakter, deshalb werden Stimmen wie Orchesterinstrumente behandelt. Lachenmann ist noch ganz dem postdramatischen Operntheater des späten 20. Jahrhunderts verpflichtet, wie es damals durch die erfolgreichen deutschen Intendanten Peter Ruzicka und Klaus Zehelein geprägt ist: Da wird keine Handlung mehr erzählt, auf konkrete Rollen wird verzichtet, Texte bleiben meist schwer verständlich, dafür werden der Musik bis anhin unbekannte Räume geöffnet, während äußerliche Theatervorgänge zugunsten der Musik zurückgenommen werden.

Schneewittchen ist ein Werk von ganz anderer Art, sowohl was seine Position innerhalb des Gesamtschaffens von Holliger angeht als auch in seinem Verhältnis zur musikhistorischen Situation am Ende des letzten Jahrhunderts. 
Holliger, der in Cardiophonie (1971), beim Magischen Tänzer (1963-65, nach Nelly Sachs) und in seinen experimentellen Beckett-Stücken (Come and go, 1976/77; Not I, 1978-80; What Where, 1988) die Grenzen des Theaters experimentell erkundete, kehrt mit dem Schneewittchen gewissermaßen zum Theater und zur Gesangsoper zurück, nicht etwa um die Operngattung zu restituieren, sondern um eine mehrschichtige theatralische Komplexität zu erreichen, die er braucht, um dem tiefgründigen Text von Walser gerecht zu werden: »Ich wollte vor allem nur durch den Lieferanteneingang hineingehen, nicht vorne durch das große Portal. Es sollte eine Musik sein, die ganz liebevoll mit dem Text umgeht . $^{38}$

\subsection{Maskenspiel und unmaskierter Prolog}

Es gibt zwei Arten von Opern: Die eine könnte man monoperspektivisch nennen, die andere polyperspektivisch. In der monoperspektivischen Oper steht hinter jeder Figur die Gedankenwelt ihres Autors. Wagner wäre dafür ein gutes Beispiel, denn die einzelnen Figuren müssen einen jeweils anderen Aspekt von Wagners Ideenwelt erklären und können sich daher aus Wagners Führung kaum herauslösen. In der polyperspektivischen Oper verschwindet der Autor im Gewimmel der Handlung und der dramatischen Konflikte. Jede Theaterfigur verfolgt ihren eigenen Weg, verkörpert eigene Perspektiven, und Librettist wie Komponist finden ihr Vergnügen darin, diese je verschiedenen Wege der Figuren so darzustellen, dass sie als Autoren gar nicht in Erscheinung treten. Ein schönes Beispiel dafür wären die autonomen und zugleich befreiten Figuren der reifen Mozart-Opern. Mozart gelingt es, in seiner Musik eine ironische Distanz zu den Figuren aufzubauen und doch als Autor unerkannt zu bleiben. Diese Autonomie führt dazu, dass keine einzige von Mozarts Figuren durchwegs böse gezeichnet wird - während es bei Wagner hoffnungslos Böse zuhauf gibt. Sogar wenn das Libretto den Bösen klar benennt, rettet Mozart die Figuren in seiner Vertonung, zum Beispiel Osmin in der Entführung aus dem Serail.

Überträgt man diese zwei Prinzipien auf Robert Walser, so wird deutlich, dass dieser bei Schneewittchen (SW 14, 74-115) die zwei Theatertypen verschränkt: Einerseits spricht in jeder Figur immer nur Walser, und jede der fünf Personen ist gleichsam Walser; andererseits benutzt Walser alle Kniffe des polyperspektivischen Theaters, um die fünf Figuren zu differenzieren (vgl. dazu die Ausführungen zu Wagners Theater bei Ruedi Häusermann in Kap. 8.5.1). Einem

38 Holliger/Zimmermann: »Ich hoffe, ich habe nie in meinem Leben etwas sver-tont «, S. 38. 
Puppenspieler ähnlich inszeniert Walser ein lautes Spektakel, um zu überspielen, dass alle Figuren von der gleichen Hand geführt und mit der gleichen Stimme belebt werden. Die reale Handlung ist mithin ein >Maskenbalk, etwas Vorgespieltes, um zu verstecken, dass hier einer eine Monoperspektive als fünffache Persönlichkeitsspaltung durchführt.

Robert Walser beginnt seine Geschichte dort, wo das Grimm'sche Märchen vom Schneewittchen endet. Alle Protagonisten leben noch zusammen und alles dreht sich immer und immer wieder um das Verbrechen von Jäger und Königin. Freuds gesamtes psychoanalytisches Werkzeug ist in diesem Stück exponiert, die Handlung mit Rollenspielen in heutigen Psychotherapien durchaus vergleichbar. Kostüme werden getauscht, alle lügen und alle sagen doch die Wahrheit, Bewusstes und Unbewusstes vermischen sich.

Das Kongeniale an diesem 1901 in einer der wichtigsten Zeitschriften der Moderne, der Insel, veröffentlichten Stück ist, dass Walser das Märchen mit der Tragödie überkreuzt:

1. Szene, Exposition: Schneewittchen erinnert an das Verbrechen. Die Königin streitet alles ab und erklärt Schneewittchen für krank. Der Jäger stimmt der Königin zu; der Prinz verteidigt Schneewittchen.

2. Szene, steigende Handlung: Der Prinz überhäuft Schneewittchen mit einem Redeschwall, bis er im Garten die Königin und den Jäger beim Sex beobachtet und völlig hingerissen ist. Sofort verliebt er sich in die Königin und verlässt Schneewittchen. Ihr Fazit: »Geh alles, wie es gehen muß.« (SW 14, 89)

3. Szene, Peripetie: Die Szene ist ein Gespräch von Königin und Schneewittchen, die Begegnung von Maria Stuart und Elisabeth I. in Walser'scher Verdrehung: Ausgerechnet Schneewittchen bittet die Königin um Vergebung und unterwirft sich ihr. Die Königin zählt nun ihre eigenen Vergehen auf, aber Schneewittchen will vergessen. Daraufhin benennt die Königin ihre eigene Krankheit: »Ich hasse mich viel mehr wie dich.«(SW 14, 94)

4. Szene, Retardierung und misslungener Lösungsversuch: Der Prinz erklärt der Königin seine Liebe. Sie weist ihn zurück und schlägt vor, die Mordszene »als ob es wirklich sei« (SW 14, 97) nochmals nachzuspielen. Diese Nachstellung kippt um in einen zweiten Mordversuch, weil die Königin aus der Rolle fällt und erneut die Ermordung Schneewittchens wünscht. Die Königin anschließend: »Wir spielten ja soeben nur! Und die Rollen standen uns gut. « ${ }^{39}$

5. Szene, Katastrophe: Nach dem gespielten Mord sind alle Figuren gebrochen - nur im Verdrängen, in der kollektiven Lüge kommen sie zusammen. Zum Schluss fordert der Jäger Schneewittchen sogar auf, die

39 So nach Holligers Partitur, S. 246f. Im Original lautet der Text: »Wir spielten ja soeben nur! Traun, und die Rollen standen gut.« (SW 14, 99) 
Königin zu küssen. Und als Deus ex machina muss der König alle beruhigen und in sein Schloss einladen.

Diesem >Ballo in maschera< stellt Holliger einen unmaskierten Prolog voran, der die auffällige Verschränkung von Poly- und Monoperspektivik explizit macht. Dieser reflektierende Rahmen ist Holliger so wichtig, dass er ihn auch im Werktitel verzeichnet: »Schneewittchen, Oper in fünf Szenen, einem Prolog und einem Epilog nach Robert Walser«. Die Zahlen Fünf und Sieben sind schon dem Werktitel eingeschrieben: fünf Szenen und zwei Rahmenteile, insgesamt sieben Teile.

Im Prolog >atmen< Orchester und die fünf Sänger gleichsam mit einer einzigen Lunge, sind ein einziges Organ, ein auskomponiertes »sch«. Aus diesem Atmen entsteht ein Unisono-Ton, das $d^{1}$. Alle Sängerinnen und Sänger singen also zu Beginn auf derselben Tonhöhe. Durch die Mischung der Lagen (tiefe Sopranstimme, gute Tenor- und Altlage, hohe Bariton- und Basslage) klingt der Ton eindringlich-befremdend. Indem dieses $d^{1}$, das bei Holliger und vielen anderen Komponisten, insbesondere aber bei Bernd Alois Zimmermann, immer wieder als Todeston fungiert, aufgespalten wird, entfaltet sich eine Polyphonie: Jede Stimme findet langsam und kontinuierlich in ihre natürliche Lage. Parallel dazu verläuft der >Geburtsvorgang` des Kammerorchesters, das den Spaltungsprozess der Singstimmen begleitet und erweitert. Es gibt keine Gestik, kein Espressivo, kein Rubato, keine individuellen Bewegungen. Der Prolog umfasst fünf $7 / 4$-Takte, die in sich isorhythmisch geordnet sind, wobei auch in dieser isorhythmischen Gestaltung Fünf und Sieben als Strukturzahlen aufscheinen. Fünf steht für die fünf Figuren des Stücks, Sieben für die Welt des Märchens, mit den im Stück abwesenden, von Schneewittchen immer wieder herbeigesehnten sieben Zwergen.

Holliger unterlegte den Prolog mit Text, der die Farbensymbolik des GrimmMärchens evoziert, und zwar mit den Adjektiven »schneeweiss « wie Schneewittchen und der weiße Sarg (Symbol für die Unschuld), »blutrot« wie das Blut (Symbol für Erotik und Sinnlichkeit) und »schwarz« wie der Sargrahmen (Symbol für den Tod).

Mit dem Prolog korrespondiert der Schluss der Oper. Im Epilog löst sich das $>$ Maskenspiek auf; es wird nur noch depraviert artikuliert. Holliger schwebte bei der Komposition dieses Epilogs ein riesiger Opernschluss im Stile von Modest Mussorgskys Boris Godunow vor, der aber wie nach innen gedreht ist und im überhellen Licht und Glanz erstickt. Ein >negativer Boris ist auch der König, der zum Schluss auftritt, scheinbar von all dem Gezänk und Gerede nichts gemerkt hat und väterlich (und >gevätterlich $<$ ) Frieden und Freude ankündigt. Dazu ertönen wie in Boris Godunow Choräle und Glockenklänge, 
aber sie sind nur als extrem gefilterte und zerbrechliche Klänge wahrzunehmen. Der Friede, zu dem Schneewittchen gerade nach der brutalen vierten Szene aufruft, ist Erschöpfung, man ist des Kämpfens, vor allem aber des Sprechens müde. »Begleitet alle uns hinein« sind Schneewittchens letzte Worte. Sie enden auf dem höchsten Ton ihrer ganzen Partie, auf dem $d^{3}$. Da ist er wieder, der Todeston $d$, diesmal aber nicht mehr in der Mitte wie im Prolog, sondern im stimmlichen Grenzbereich eines Soprans - und bei Holliger muss die Sopranistin diesen anstrengenden höchsten Ton am Ende der Oper und nach einer riesigen Partie noch erreichen können. Die psychophysischen Verspannungen, die er vor allem in seinen wilden Jugendjahren so liebte, sind auch hier noch immer vorhanden.

\subsection{Grundprinzip Als-ob}

Nach dem Prolog beginnt die eigentliche Oper aggressiv und wie ein Schock. Alle Beteiligten ziehen sich nun gleichsam Masken vors Gesicht. Königin und Schneewittchen wirken maskiert in ihren Kostümen und Haltungen, aber auch die Musik von Holliger wechselt nach dem statischen und bewegungslosen Prolog sofort in ein maskierendes Verhalten: Begleitet von aufsteigenden Quarten in den Hörnern und einer >Mannheimer Rakete < in der Harfe, beginnt die Königin mit einem scharfen »Sag', bist du krank?« Bei Schneewittchens Antwort ändert die Klangwelt abrupt, es spielen Celesta, hohe Streicher und Flöte. Trotz diesem plötzlichen Wechsel der Instrumentierung antwortet Schneewittchen mit ähnlichem intervallischem Material wie die Königin, allerdings in einer Umkehrungsfigur: Der Frage der Königin, die wie ein Befehl vertont ist, folgt Schneewittchens Antwort wie eine schüchterne Frage.

Mit solcher Drastik etabliert Holliger von allem Anfang an das Grundprinzip der Oper: Weder musikalisch noch textlich ist hier irgendetwas so zu nehmen, wie es dasteht. Hinter jeder Geste, hinter jedem Wort und jedem Ton steht ein Anderes, welches diesen Ton als ein Als-ob ausweist - ein Als-ob, das in der Handlung ständig präsent ist und in der vierten Szene seinen Höhepunkt erreicht, wenn der Jäger Schneewittchen noch einmal ermorden will, aber nur zum Spiel und als ob.

Dieses Prinzip des Als-ob wendet Heinz Holliger mit verschiedenen Mitteln an. Zentral ist dabei eine genaue, quasi wörtliche Vertonung des Textes. Holliger hat dafür ein reiches Repertoire an Tonzeichen und musikalischen Figuren entwickelt, die mit den entsprechenden Wörtern und Textstellen bzw. den Theaterrollen korrespondieren. Schon der erwähnte Anfang zeigt, dass die 
sadistische und herrschsüchtige Seite der Königin mit aufstrebenden Quarten, mit Blechblasinstrumenten, Marimba (als einer Art modernem Knocheninstrument), Harfe und Pizzicati gekennzeichnet ist; Schneewittchens Instrumente sind die Flöten, Glasharmonika, Celesta und die hohen Streicher. Aber je nachdem, wer was sagt, können diese Zuordnungen wechseln. Wenn Schneewittchen später die Rolle der Königin übernimmt, erklingen auch bei ihr die Instrumente der Königin.

Ein anderes Mittel sind die trügerischen Fährten, die Holliger legt, indem er >falsche< Bezüge herstellt. So wie sich Walser in seinen Texten jederzeit mehr oder weniger deplatzierte Exkurse herausnimmt, die zuweilen auf bloßen Assonanzen beruhen und nur über weit entfernte Assoziationsketten mit dem Haupttext in Verbindung gebracht werden können, so erlaubt sich Holliger vergleichbare Abschweifungen. Da wird die »schlammige Flut« oder der »Tau« mit Wassergong begleitet und die »Meute Hunde« mit Hörnergebrüll beantwortet. Da werden absichtlich Umdeutungen vorgenommen, sodass das Wort»Stickerei« zu >erstickten〈Klängen in der Musik führt, bei »Wellenschlag « der metrisierte Rhythmus aussetzt oder das Wort des Prinzen »als Freier« im Orchester mit übertriebenem liberamente-Spiel beantwortet wird. Wenn der Prinz Luft holt, wird er dabei von einer Windmaschine oder pustenden Posaunen verstärkt. Diese Assoziationen lenken in falsche Richtungen, sind bewusst gesetzte irreführende Fährten.

Holliger führt auch komische, ironische, groteske und grobe Elemente ein, am deutlichsten in der Partie des Prinzen, der herumschreit und wichtigtut, obwohl er eigentlich nur ein kleiner Knabe ist, der Schneewittchen nicht einmal geküsst hat. Hier spart Holliger nicht mit Komik und Übertreibung. Damit komponiert er in Schneewittchen auch gegen das Pathos an, mit dem man Walsers Lebenslauf zunehmend betrachtete und dem Holliger in Beiseit selbst gefrönt hat - und es zum Schluss auch in Schneewittchen tut. Da geht die Musik durchaus nicht »ganz liebevoll mit dem Text um«, wie es Holliger sagt (vgl. Anm. 38), sondern zuweilen auch obszön-grobschlächtig, zum Beispiel wenn beim Sex zwischen Jäger und Königin zum Wort »Liebeswonn!« der Schlagzeuger auf dem tambour à corde ein >Löwengebrüll< loslässt, oder wenn beim Als-ob-Mord an Schneewittchen Amboss, Peitsche, Ratsche und Tamtam einen höllischen Lärm in Kanonform veranstalten. Solche Bezüge zwischen Text und Musik könnte man insofern als >negative Übersetzungen< bezeichnen, als sie den Text teilweise zerstören bzw. die Figuren böser oder unbeholfener machen, als sie es bei Walser tatsächlich sind. Zuweilen scheint es, etwa bei der >bösen Zeichnung der Königin, als würde der Komponist hier wie ein kleines Kind im Puppentheater seine ganze Antipathie gegen die herrschsüchtige Frau ausleben. 


\section{9 $\quad$ Ein musikalisches Labyrinth}

Eine weitere Ebene von Holligers Schneewittchen sind Zitate und Anlehnungen aus der ganzen Musikgeschichte. Diese beziehen sich zum Teil sehr konkret und direkt auf das Libretto: Bei den Worten »bring ihn wieder« zitiert Holliger im Orchester zum Beispiel Fuchs, du hast die Gans gestohlen. Oder wenn die Königin in der ersten Szene Schneewittchen auffordert, sie solle sich wie ein »Schmetterling « bewegen, erklingt in der Celesta die Klavierbegleitung des bereits erwähnten Liedes Schmetterling nach Morgenstern, das Holliger mit siebzehn Jahren seiner Mutter - an Walsers Todestag - zu Weihnachten schenkte.

Auch diese Zitate sind falsche Fährten, die mit dem, was eigentlich abläuft, wenig zu tun haben. Gerade in der Schmetterling-Szene saugt die Königin Schneewittchen wie ein Vampir aus. Eine falsche Fährte ist es auch, wenn Holliger Le rossignol en amour von François Couperin in dem Moment zitiert, als der Prinz Schneewittchen mit Reden überhäuft, um sie nicht lieben zu müssen, und in den höchsten Tönen von der Nachtigall wispelt.

Die ganze Partitur ist durchsetzt von solchen Zitaten oder Beinahe-Zitaten. Diese werden umso offensichtlicher, je deutlicher auch Walsers Text sich zitathaft gibt oder an Liedhaftes anspielt. So vertont Holliger die Liebeserklärung des Prinzen an die Königin in einer Weise, dass das durchaus falsche Pathos der Königin als Kirchenchoral und die schüchterne Gestelztheit des Prinzen als kleines Kinderliedchen daherkommt. Überhaupt sind häufige Anklänge an Volksgut hörbar, Liedchen und Tänze; zuweilen sind es auch Zitate von Zitaten, etwa Maries Wiegenlied aus Alban Bergs Wozzeck, das rhythmisch und melodisch aufscheint und zu einer Art Grundtopos der Schneewittchen-Partie wird.

Diese Anspielungen zeigen, dass Holliger die Zitate nicht nur als falsche Fährten verwendet, sondern sie zuweilen die Sache selbst mit äußerster Präzision benennen. So entstehen musikhistorische Querbezüge, die hinter der Schneewittchen-Figur eine ganze Reihe von Figuren und Werken aufscheinen lassen:Marie aus Bernd Alois Zimmermanns Soldaten, Marie aus Bergs Wozzeck, Genoveva aus Schumanns gleichnamiger Oper, die von Holliger immer wieder konzertant aufgeführt wurde, aber letztlich auch Bachs Johannes-Passion. Ein weiteres Beispiel für ein komplexes Zitat sind Schumanns Märchenbilder die bei den Worten »das Märchen lügt « verwendet werden. Dieses Zitat ist nur schwer erkennbar, da Holliger die Melodie perfekt in den musikalischen Kontext einarbeitet. Überhaupt entscheidet bei den Zitaten der Grad der Markierung über die Eigentlichkeit des Verweises: Wenn das Zitat uneigentlich gemeint ist und in die falsche Richtung lenkt, wird es von Holliger klar markiert; wenn es die 
eigentliche Sache trifft, ist es weitgehend in die Textur eingebunden und als solches bestenfalls als leise Assonanz wahrnehmbar.

\subsection{Kanon, Choral, Palindrom, Chiasmus}

Die musikalischen Grundformen des Scardanelli-Zyklus, nämlich Kanon und Choral, sind auch in Schneewittchen prägend. Mit der Kanon-Technik kann Holliger Strukturen multiplizieren und in der Multiplikation verwischen. Häufig verwendet er Kanon-Techniken auch in der Variante der Engführung, das heißt mit einander schnell folgenden Einsätzen. Auch der Kanon steht in Bezug zu Walsers Text. Hätte Walser komponiert, wäre der Kanon wohl sein favorisiertes Verfahren gewesen, arbeitet seine Literatur doch immer wieder mit der Selbstähnlichkeit von Worten und perpetuiert sich zuweilen in Klangund Bedeutungsketten.

Der Kanon setzt Selbstähnlichkeit in horizontaler Richtung in Gang. Selbstähnlichkeit in vertikaler Richtung führt zum zweiten wichtigen Gestaltungsmittel in Schneewittchen, nämlich zum Choral. Im Epilog etwa endet die Oper in strengen Choralvariationen, die einen himmlischen und wie hinter Glas fast unhörbar jauchzenden, zugleich aber innerlich erstarrten Klang erzeugen, mit dem Holliger auch den literarisch verstummten Walser der Herisauer Zeit evozieren wollte.

Es gibt in Schneewittchen einen Moment, in dem die vertikale Orientierung des Chorals und die horizontale des Kanons sich in extremer Weise überkreuzen: an der Stelle nach dem Als-ob-Mord an Schneewittchen, wo die Königin singt »Wir spielten ja soeben nur! Und die Rollen standen uns gut.« Hier lagert Holliger in einem 7/8-Takt - eine weitere strukturelle Anspielung an die sieben abwesenden Zwerge - in allen Instrumenten verschiedene Motive der Oper übereinander und erreicht damit vertikal wie horinzontal den höchsten Dichtepunkt des gesamten Werks (vgl. Abb. 22). Das einzige eigentliche Tutti der Oper besteht in der größtmöglichen Aufspaltung, ja Zerrissenheit des Orchesters. Auch dieser Takt ist übrigens eine Reminiszenz an Bergs Wozzeck, wo nach Maries Ermordung ebenfalls alle ihre Motive synchron erklingen.

Eine Steigerung der Selbstähnlichkeit sind die musikalischen Palindrome, die von vorwärts und rückwärts bzw. von oben und unten gleich aussehen. Solche Palindrome durchziehen die ganze Oper. Auch in Walsers Schneewittchen wimmelt es von Beinahe-Palindromen wie »End küßt sich in dem End, wenn auch Anfang noch nicht zu Ende ist «(SW 14, 110), ein Satz, der von Holliger mit zwei Palindromschlaufen vertont wird. Ein besonders eindrückliches Beispiel 


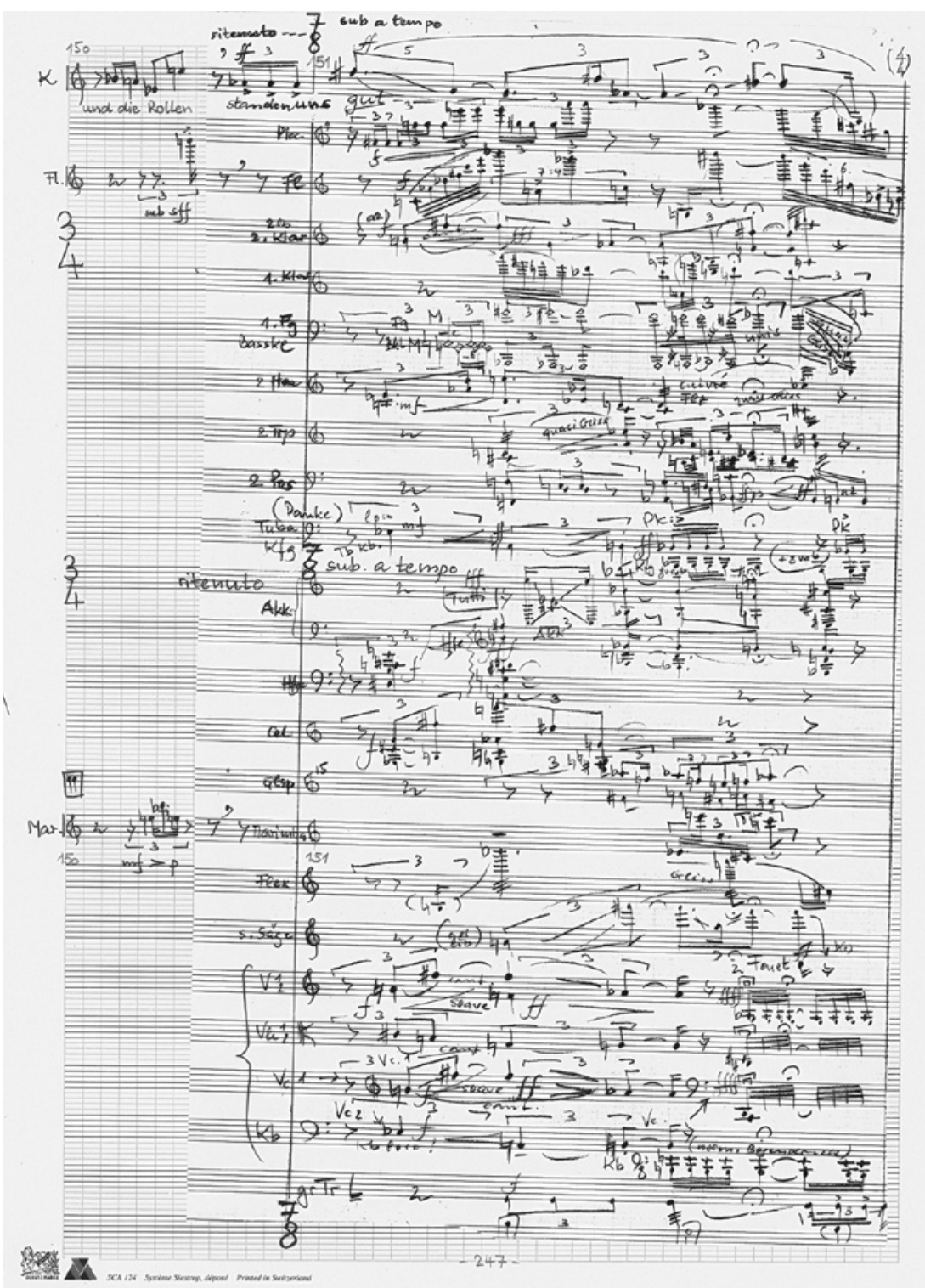

Abb. 22

Heinz Holliger: Schneewittchen, IV. Akt. S. 247, T. 15 of. 
findet sich in Schneewittchens Aria in der zweiten Szene, die eine ähnliche Funktion einnimmt wie das Lied der Lulu in Alban Bergs gleichnamiger Oper. Dieses Lied, dem Berg die höchst auffällige Tempoangabe »im Pulsschlag der Sängerin « beifügt, ${ }^{40}$ endet mit der palindromischen Essenz, zu der Wedekind Lulus Wesen verdichtet: »Ich habe nie in der Welt etwas anderes scheinen wollen, als wofür man mich genommen hat, und man hat mich nie in der Welt für etwas anderes genommen, als was ich bin. ${ }^{41}$ Holliger setzt nun in der Aria einen Satz ins Zentrum, der an Wedekinds Palindrom erinnert: $\gg$, ich verlange ja nichts mehr, als daß ich lächelnd tot bin, tot. Das bin ich auch und war es stets.«(SW 14, 85)

Die Gegenform des Palindroms ist der Chiasmus, der die Elemente über Kreuz einander gegenüber ordnet. Liegt beim Palindrom eine extreme Umkehrbarkeit einer Gestalt vor, so stellt der Chiasmus die größtmögliche Nicht-Umkehrung dar. Auch diese chiastischen Formen benützt Holliger in Scheewittchen häufig: In derselben musikalischen Gestalt wird von extrem leise zu extrem laut gewechselt, von Stopfung oder Dämpfung zur Öffnung des Klangs, von Höhe zu Tiefe, von Feinheit zu Grobheit, von kurz zu lang etc., wobei sich diese Parameter gegenseitig überkreuzen können. Auch der Chiasmus ist ein beliebtes Stilmittel von Walser, das sich in der Sprache wie in den Figuren-Konstellationen wiederfindet.

\subsection{Gesangsoper}

In Schneewittchen sind die Singstimmen vom Orchester abgesetzt. Sie gehören einer anderen Welt an als das Orchester und tragen - das wäre eine Gemeinsamkeit mit Mozarts, aber auch Bergs und Schönbergs Opern - bereits die musikalische Substanz in sich. Das Orchester betreibt einen enormen Aufwand mit viel Material, greift oft lautstark ein, wird manchmal zum Partner und antwortet den Sängern, übernimmt Doppelgänger-Funktion, geht an einigen Stellen auch einen eigenen Weg - die eigentliche Verbindlichkeit der Musik liegt jedoch in den Singstimmen. Die melodische Qualität der Singstimmen unterscheidet Schneewittchen von vielen modernen Rezitativopern. Deren Modell orientiert sich letztlich immer noch an Wagner, indem eine wenig

40 Der den Taktschlag gebende Dirigent ist damit außer Kraft gesetzt. Heinz Holliger gibt im Scardanelli-Zyklus dieselbe Anweisung den Sängerinnen und Sängern bei den SOMMERTeilen, um komplexe kanonische Überlagerungen zu ermöglichen und das gemeinsame Zeitgefühl aufzuheben.

41 Wedekind: Der Erdgeist, S. 207. 
konturierte Textrezitation von einer dominierenden Orchester-sinfonie< begleitet wird. Wer in Holligers Schneewittchen nach solchen sinfonischen Prinzipien sucht, wird enttäuscht. Auch wenn sich das Orchester zuweilen drastisch in den Vordergrund drängt, spricht es eigentlich immer durch die Stimmen hindurch und entwickelt keine Autonomie im Sinne eines sinfonischen >Wir-Effekts $<$. Deshalb verzichtet Holliger im Orchester konsequent auf das Tutti und beschränkt sich über weite Strecken auf unterschiedliche und ständig wechselnde Klangausschnitte. Wie in der barocken Oper hat Holliger ein ganzes Spektrum an >Continuo<-Instrumenten eingeführt: Harfe, Celesta, Akkordeon, Marimba, Vibraphon und Glasharmonika. Gerade die Glasharmonika wird so etwas wie der Hauptton der Oper und färbt mit ihrem zarten, aber durchdringenden Klang die musikalischen Ereignisse ein. Dabei ist sie immer mit der Welt von Schneewittchen assoziiert. Schlagzeug, Kontrabass, Posaunen und Tuba werden häufig als Zerrspiegel der Gesangspartien verwendet und rumpeln grotesk und gespenstisch zum überspannten Gesang.

Wenn man bei Wagner anmerken könnte, dass die Gesangsstimmen erst durch das Orchester ihre Bedeutung bekommen, so ließe sich bei Holliger das Gegenteil feststellen: Die Gesangsstimmen hauchen dem wilden Beziehungsund Verweisgestrüpp des Orchesters Leben ein, geben den drastischen >Übersetzungen< ihren Sinn, verstärken damit aber nur die gespenstische Wirkung dieser absonderlichen Begleitkapelle. Mit dieser >Gesangsoper<, bei der die Singstimme Ausgangspunkt und Zentrum bildet, markiert Holliger am Schluss des 20. Jahrhunderts etwas durchaus Neues und Einmaliges in der Operngeschichte, ${ }^{42}$ insofern als sich Schneewittchen von den zahlreichen neoromantischen Literaturopern ebenso absetzt wie von jener Tradition, in der auch Lachenmanns Mädchen mit den Schwefelhölzern steht, die die Oper zunehmend mit absolut-musikalischen Ansprüchen entdramatisiert, auf Programmatisches und Illustrierendes verzichtet und - mit strengen formalen und strukturellen Prinzipen - Oper wie eine komplexe Sinfonie komponiert.

Mit ein Grund für die Bedeutung der Singstimme könnte in Holligers zweiteiligem Kompositionsverfahren liegen, ${ }^{43}$ das er in Zusammenhang mit Walsers Schreibverfahren brachte. Holliger skizzierte in einem ersten Schritt nur die Singstimmen und arbeitete die Komposition anschließend aus. Robert Walser entwickelte, wie er in einem Brief an Max Rychner bemerkt, ein eigentümliches »Bleistiftsystem, das mit einem folgerichtigen, büreauhaften

42 Interessanterweise unternahm Johannes Fritsch ein paar Jahre früher bei Aschenbrödel einen ähnlichen Restitutionsversuch der Gesangsoper (vgl. Kap. 13.3).

43 Zu Holligers zweiphasigem Kompositionsverfahren vgl. Stenzl: »Wahn, alles Wahn ...!«, S. $75^{-8}$ o. 
Abschreibesystem verquickt ist « (BA 2, 299). Wahrscheinlich ab 1917 notierte er nach einer Schreibkrise in einem ersten Schritt die Texte mit Bleistift in Kleinstschrift - die bekannten Mikrogramme -, um sie dann nochmals in normaler Größe und mit zahlreichen Variationen abzuschreiben. Im Brief an Rychner erklärt Walser, er habe angefangen, »zu bleistifteln, zu zeichnelen, zu gfätterlen. Für mich ließ es sich mit Hülfe des Bleistiftes wieder besser spielen, dichten; es schien mir, die Schriftstellerlust lebe dadurch von neuem auf. [...] [B] eim Abschreiben aus dem Bleistiftauftrag lernte ich knabenhaft wieder schreiben.« (BA 2, 299f.) Das entspricht in vielen dem, was die Beschäftigung mit Robert Walser bei Holliger ausgelöst hat. Anlässlich der Zürcher Uraufführung von Schneewittchen 1998 sagt Holliger dazu:

Ich fühle, dass ich durch ihn [Walser] in ganz andere Regionen geschubst werde, dass er mich, der ich oft als Lamentoso-Musik-Spezialist bezeichnet werde, in eine Sphäre von unglaublicher Leichtigkeit hineinbläst und dass ich mich dabei auch neu mit meinem eigenen Leben beschäftige. Jugendgedanken tauchen wieder auf, das ganze Leben geht wie ein Fächer auf. ${ }^{44}$

Während für Walser »der Prozeß der Schriftstellerei naturgemäß eine beinahe in's Kolossale gehende, schleppende Langsamkeit«(BA 2, 299 $)^{45}$ darstellt, versucht Holliger mit seinem Entwurfsverfahren gerade schneller zu werden:

Ich bin fast eifersüchtig auf Maler, die - wie Picasso - manchmal wahnsinnig schnell malen, aber immer das ganze Bild vor sich haben und sehen: »Wo muss ich noch etwas dazu machen? « [...] Das Schreiben von Musik ist so unendlich langsam im Vergleich mit der Intuition, mit der man Musik innerlich hört. Das ist wie ein Gedankenblitz, man kann innert weniger Sekunden ein einstündiges Stück denken. [...] Ich wollte - indem ich möglichst schnell notiere - diesen Blitz ein bisschen verlängern, damit er mir leuchtet, solange ich mein Gedächtnisprotokoll schreibe. ${ }^{46}$

Oft komponiert Holliger vergleichsweise sehr rasch. So schrieb er während der Holliger-Walser-Woche 1996 in Biel, die von Proben, Konzerten und Kursen vollgestopft war, zur Hochzeit von Sabine Gertschen und Elmar Schmid am 21.12.1996 (man beachte das verspiegelte Datum 21.12.!) Ein Klar- und bassklari-nettliches Zwiegesängelchen für Sabine und Elmar. ${ }^{47}$ Mit dem ihm eigenen

44 Holliger/Meyer: Über Robert Walser und »Schneewittchen«, S. 8.

45 Zur Mikrogrammschrift von Robert Walser vgl. Morlang: Melusines Hinterlassenschaft, Walt: Improvisation und Interpretation.

46 Holliger/Zimmermann: »Ich hoffe, ich habe nie in meinem Leben etwas sver-tont «, S. 31.

47 Nach dem Gedicht Es ist Nacht (SW 13, 30). Der im Titel abgesetzte Wortteil »-nettliches« bezieht sich auf Mischa Käsers Nettchen (vgl. Kap. 8), das während 


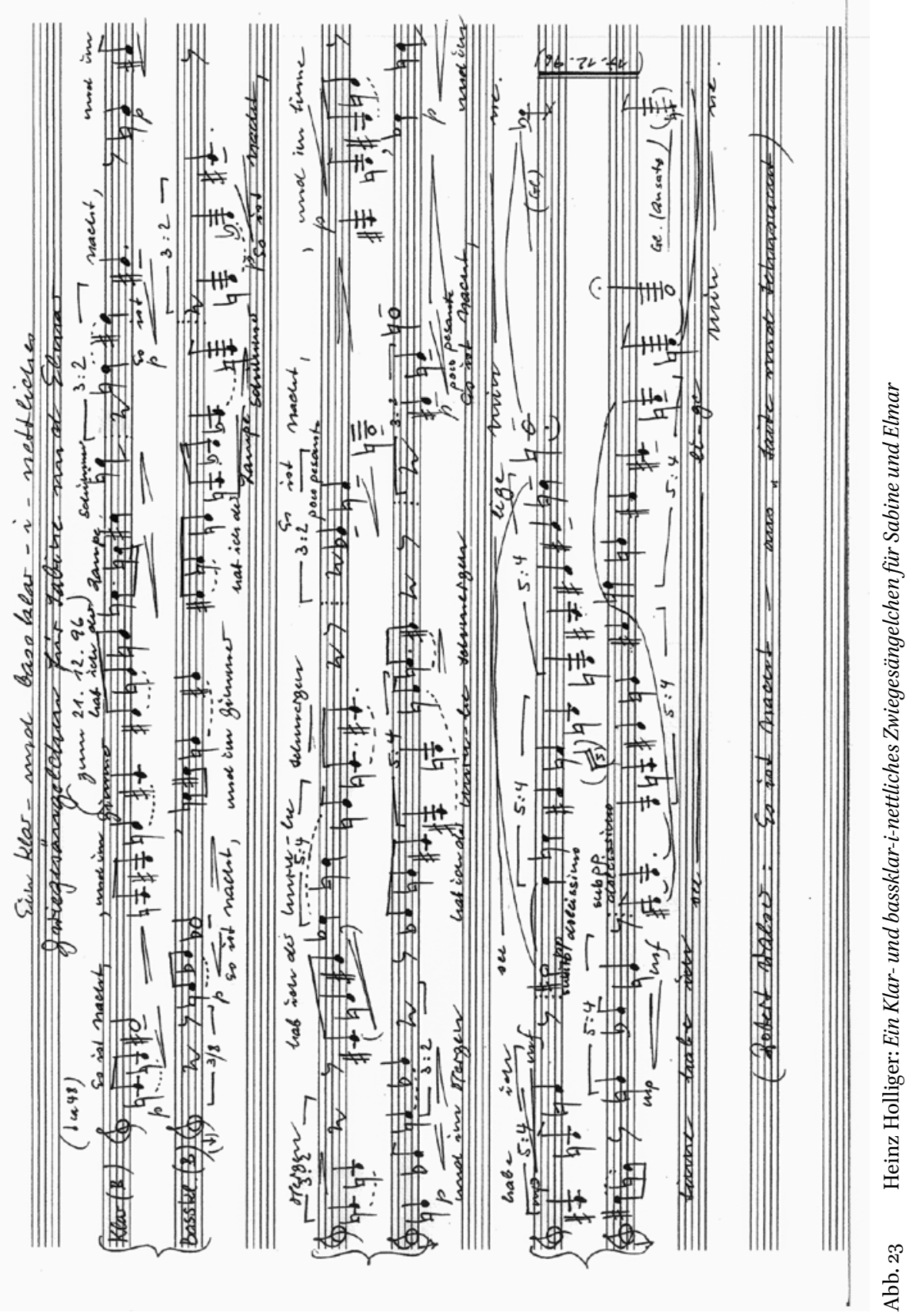


schalkhaften Lächeln zeigte er mir am Ende dieser Woche dieses rasch hingeworfene Duett; ein Spiegelkanon als doppelter Kontrapunkt und ein stummes Melodram (vgl. Abb. 23) ${ }^{48}$ Man könnte lange über das Beziehungsideal hinter diesem $>$ Zwie $<-G e s a n g$ meditieren, hier nur ein paar Stichworte: völlige Autonomie der Stimmen, kaum gemeinsam einsetzende Töne, alles streng aufeinander bezogen und ineinander gespiegelt, reiche Harmonik wegen konsequenter Gegenbewegung und - im stummen Melodram - viel Unausgesprochenes, Gedachtes, >Unveröffentlichtesく.

\subsection{Die bisherigen Aufführungen}

Im Gegensatz zu Helmut Lachenmanns Mädchen mit den Schwefelhölzern, das seit der Uraufführung schon einige Male nachgespielt wurde, gibt es von Holligers Schneewittchen bisher nur zwei, allerdings vielbeachtete Produktionen. Beide waren von herausragender musikalischer Qualität unter der Leitung des Komponisten, hinterließen aber einen zwiespältigen Eindruck in szenischer Hinsicht. In beiden Produktionen hat die Inszenierung die musikalischen Differenzierungen der Partitur und die feinen Netzwerke zwischen Kammerorchester und Solostimmen überdeckt. Die Uraufführungsinszenierung von 1998 verantwortete der Schweizer Regisseur Reto Nickler. Sie hatte den Vorteil, dass die szenischen Hauptzüge nachvollziehbar waren; auch das Maskenhafte wurde mit teilweise monumentalen Kostümen gut demonstriert. Allerdings entstand eine Diskrepanz zwischen dem kleinen Orchester und dem großen Bühnenraum, sodass die Miniaturisierungen der Musik in der Inszenierung wenig Echo finden konnten. ${ }^{49}$

Von einem schon fast grotesken Missverständnis war die Basler Produktion mit dem Regisseur, Maler, Bühnen- und Kostümbildner Achim Freyer geprägt. Freyerließsich nämlich gar nicht auf die Partitur ein, erzählte auch nichtWalsers Geschichte nach, sondern entwarf ein eigenes Stück: In der Vorbereitung

der Holliger-Walser-Woche die Vor-Uraufführung erlebte und in dem Elmar Schmid die musikalische Leitung innehatte. Der Grund für die im Gedicht angesprochenen >Schmerzen<, die nicht gerade zu einem Hochzeitsständchen passen, ist ein naher Todesfall in der Familie Schmid, der sich während der Holliger-Walser-Woche ereignete.

48 Eine Aufführung als tatsächliches Melodram ist denkbar: »Heinz [Holliger] sagt, Rezitation sei möglich, aber leise und diskret, und keinesfalls etwas Gesungenes ...«. E-Mail von Barbara Golan (Agentin von Heinz Holliger) an Roman Brotbeck vom 12.10.2020.

49 Bei der ähnlich kammermusikalisch angelegten Oper Lunea von Heinz Holliger (2018) hat Andreas Homoki die Bühne des Opernhauses Zürich auf ein Kleintheater reduziert und damit inszenatorisch und musikalisch eine viel größere Übereinstimmung erreicht. 
sammelte Freyer zentrale Wörter des Librettos und fügte diese zu einem Wortkatalog zusammen. Er teilte diesen Katalog in sieben Abschnitte ein und gestaltete aus den Wörtern dieser jeweiligen Abschnitte je sieben unterschiedliche Theaterräume, die jeweils fünf Minuten dauerten, und zwar unabhängig von den Szenen des Librettos und den musikalischen Zwischenspielen. ${ }^{50}$ Für diese Räume entwickelte Freyer je eigene Bewegungsabläufe und -zeiten, auch den einzelnen Rollen sind verschiedene Tempi zugeordnet, sodass nur die sichtbaren Bühnenarbeiter in normaler Alltagszeit spielen und arbeiten. Weil im Libretto das Wort \Schnee< eines der häufigsten Wörter ist, entschied der Regisseur, die Räume jeweils mit zweiminütigen Schneeszenen zu unterbrechen bzw. zu verbinden. Mit diesen sieben, jeweils exakt fünf und zwei Minuten dauernden szenischen Räumen schaffte Freyer eine metaphysische Assoziations- und Bilderwelt: Traumhaftes, Märchenhaftes und pittoreskes Puppenspiel mischt sich mit Beängstigendem und Schockierendem. Das ImKreise-Drehen der Walser'schen Figuren wird imitiert, indem jeder dieser sieben Räume im Laufe des Opernabends mindestens zweimal erscheint - wie ein Karussell von Walser-Welten.

In der Tradition seines einstigen Vorbilds Ruth Berghaus, für die er in seiner DDR-Zeit als Bühnen- und Kostümbildner tätig war, inszenierte Achim Freyer also nicht die Geschichte. Das ergab eine höchst attraktive Bühnenwelt, auch deshalb, weil sich weit mehr maskierte Figuren auf der Bühne herumtummelten, als sie im Stück von Walser vorkommen. Dieser Bilderreichtum hatte nur einen entscheidenden Haken: Mit Holligers Partitur oder Walsers Dramolett hatte das alles eigentlich nichts zu tun. Es war ein autoritäres Konzept, das der Partitur übergestülpt wurde und das die freie Fantastik von Walser und Holliger mit einer uniformen Stoppuhr-Ästhetik in gleich große bzw. kleine Stücke zerschnitt - in der Hoffnung, es würde sich zum Schluss ein höherer Sinn ergeben.

Spannend ist, dass Freyer 1997 auch die Uraufführung von Lachenmanns Mädchen mit den Schwefelhölzern verantwortete und dort sehr viel mehr überzeugte, weil Lachenmanns viel abstraktere Musik zu Freyers Inszenierungskonzept besser passte.

Dass weder die handwerklich gut gebaute realistische Umsetzung von Reto Nickler noch die extreme Regietheatervariante Freyers der Oper von Holliger wirklich gerecht wurden, liegt aber auch am Werk selber. Dieses ist so sehr mit Inhaltlichem aufgeladen, so sehr immer auch Reflexion von Oper, dass der institutionelle Raum der Oper womöglich der falsche Ort dafür ist. In frühen Gesprächen zu Schneewittchen erzählte mir Holliger, dass ihm ein 
Miniaturtheater vorschwebe, eine Art Puppentheater, in dem die Figuren auf kleinem Raum wie eingeschlossen seien. ${ }^{51}$ Während der Basler Proben zu Schneewittchen sagte er zu Heidy Zimmermann: »Ich könnte mir eine Bühne fast ohne Ausstattung vorstellen, nur weiß und fast nur Schattentheater, so dass der Klang das Bühnendekor wäre. [...] Aber Achim Freyer platzt fast vor Imagination ...« ${ }^{52}$

Vielleicht sollte man diese Idee weiterdenken und das Orchester mit seinen vielen semantisch aufgeladenen Instrumenten ins Zentrum stellen, während die Sängerinnen und Sänger sich dazwischen frei bewegen. Das würde bedeuten, das Werk mit den Mitteln des Théâtre musical aufzuführen, auf große Theaterwirkungen zu verzichten und die vielen kammermusikalischen Verhältnisse zwischen den Singstimmen und einzelnen Instrumenten punktgenau zu visualisieren. Auf diese Weise ließen sich die vielen Geschichten, die Walser und Holliger zusammenfabulieren, plastischer vermitteln.

$5^{1} \quad$ Georges Aperghis hat dieses Puppentheater 2006 mit Zeugen realisiert (vgl. Kap. 7).

$5^{2}$ Holliger/Zimmermann: »Ich hoffe, ich habe nie in meinem Leben etwas sver-tont «, S. 35 . 